\title{
Energy calibration of thin plastic scintillators using compton scattering of gamma rays
}

\author{
Wooram Cho* \\ Department of Physics, Yonsei University, Seoul, Korea \\ E-mail: wrcho@yonsei.ac.kr \\ Youngjoon Kwon \\ Department of Physics, Yonsei University, Seoul, Korea
}

\section{Myoungjin Hwang}

Sorabol High School, Seoul, Korea

\begin{abstract}
We are testing a plastic detector prototype to observe cosmic rays of $\mathrm{GeV}$ level. The energy deposit in the detector is important to know the composition of the particles which reach on the ground. We use compton scattering of RI to calculate the energy deposit of thin plastic scintillators. The experimental values are compared with Geant 4 simulation results. Polystyrene based scintilltor of $1 \mathrm{~cm}$ thickness is connected to PMT and the DAQ device including an $100 \mathrm{MHz}$ oscillator. An analog waveform is converted to 1024 FADC counts. Prototype system consist of two scintillators and their energy deposits is investigated for several different arrangements. In this proceeding, we will show the result of energy calibration of thin plastic scintillators using RI and simulation.
\end{abstract}

35th International Cosmic Ray Conference - ICRC2017

10-20 July, 2017

Bexco, Busan, Korea

${ }^{*}$ Speaker. 


\section{Introduction}

We are testing detector prototypes to detect cosmic rays of $\mathrm{GeV}$ energy level and to measure cosmic ray flux. The plate detector is thin and the material is plastic[2]. This scintillators are easy to count passages of particles such as muons. However, we still can not accurately calculate the energy that the particles leave when they pass through scintillators. At present, it is difficult to distinguish the energy or kind of the detected particles, so it is necessary to quantify the energy left by the particles passing through the plastic scintillator. Since we can get a relative value that is proportional to energy, we want to convert the relative value using a source whose energy is known as the actual value. In the case of plastic scintillators, it was found that calibration using RI was possible[3, 4]. We will investigate the reaction between RI and the plastic scintillator.

\section{Expermiments}

\subsection{Hardware}

The equipment consists of scintillators, PMTs, a DAQ device, a GPS antenna and a personal computer. The photons left by the cosmic ray particles passing through the scintillator are amplified by PMT. After the DAQ device processes the signal by the trigger logic, the waveform and arrival time data are stored in the personal computer[1].

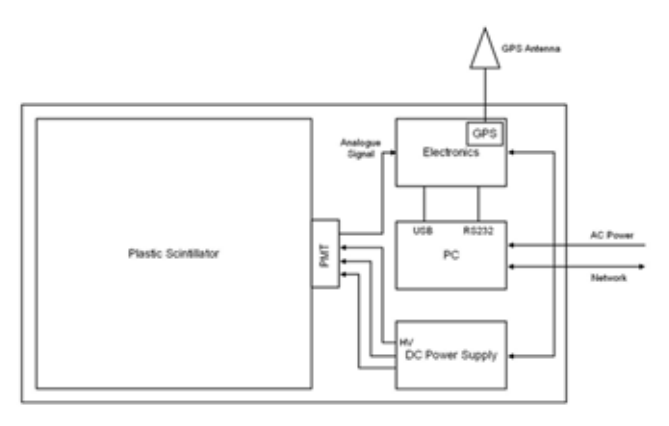

Figure 1: Model of the cosmic ray detector

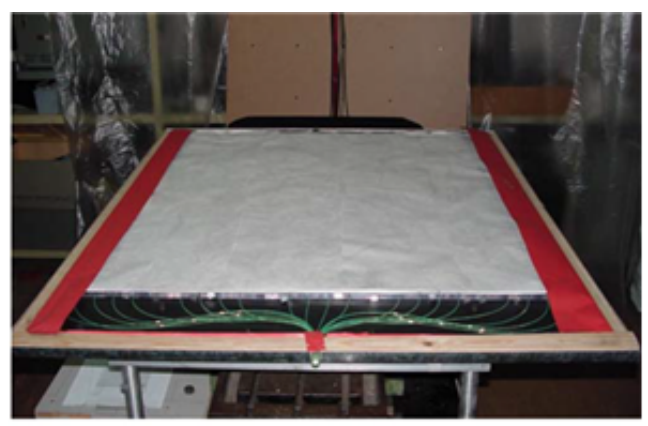

Figure 2: The scintillator and obtical fibers

\subsubsection{The scintilltor and the PMT}

Figure 2 is a picture of the scintillator. As charged particles pass through the scintillator, they lose energy and excite atoms in the scintillator. Excited atoms emit photons. Emitted photons are transmitted to the PMT through optical fibers and converted to electirc currents.

One of most widely used materials for scintillators is a plastic. A plastic has an advantage of detecting very fast decay signals of 2 to $3 \mathrm{~ns}$. For this experiment, $1000 \mathrm{~mm} \times 1000 \mathrm{~mm} \times 10 \mathrm{~mm}$ polystyrene scintillator plates are used. $194 \mathrm{~mm} \times 194 \mathrm{~mm} \times 5 \mathrm{~mm}$ units are arranged in $5 \times 5$ plates to form one layer. The scintillator used in the experiment consists of two layers. There are grooves between two layers, and 22 WLS (Wave Length Shifter) fibers of $1 \mathrm{~mm}$ diameter are inserted. Each fiber end was treated with an aluminum mirror to prevent a photon loss. Photons generated from the scintillator are transmitted to the PMT along optical fibers. 


\begin{tabular}{cc}
\hline Base materials & Polystyrene \\
Decay time & $2 \mathrm{~ns}$ \\
Dimensions & $1000 \mathrm{~mm} \times 1000 \mathrm{~mm} \times 10 \mathrm{~mm}$ \\
Light guide & 22 Y-11 type WLS-fibers of $1 \mathrm{~mm}$ diameter \\
Wavelength of maximum output & $420 \mathrm{~nm}$ \\
Fiber light attenuation length & about $330 \mathrm{~cm}$ \\
\hline
\end{tabular}

Table 1: Characteristics of the scintillator

The PMT is FEU-115M manufactured by MELZ of Russia. A length of FEU-115M is $107 \mathrm{~mm}$ and a diameter is $30 \mathrm{~mm}$. FEU-115M features a high gain and a low dark current, has a 12-stage Al-Mg alloy dynode linear focused system and a translucent Sb-K-Na-Cs photo-cathode[2].

\subsubsection{The DAQ device}

The DAQ device can process three channels simultaneously. A FPGA chip was used to identify a coincidence of 3 channels. If a signal from the PMT has a pulse height greater than threshold value, a signal is stored according to the trigger mode. The trigger mode used in this experiment is when two channels or more of three channels have a pulse height greater than threshold value. A pulse height of an analog signal is digitized in 1024 steps. Time is digitized using an $100 \mathrm{MHz}$ clock up to 950ns. Digitized signals have waveform and time information obtained from the GPS and measured with an $100 \mathrm{MHz}$ oscillator.

\subsection{The data acquisition}

For each station, LabWindows/CVI-based software running on Windows OS is used for DAQ. This program can adjust the trigger mode, threshold value, the number of events, etc. As a first step, the threshold and the trigger mode seprate cosmic rays entering each station from the background noise. ADC value of each channel is received from the DAQ device through an USB port.

\subsection{Method}

The experiment proceeded indoors. The place was a first floor laboratory of a five-story building. The scintillators of the two plates were stacked and used. The trigger mode is used to record the signal when only one of them was detected. The RI was Co60 of about $1 \mu \mathrm{Ci}$. The arrangement of the plates was tested in the horizontal and vertical arrangement, in which the Co60 was placed in the middle between the plates. The arrangement of the scintillator and the RI is shown in the Figure 3 and 4 . We measured the energy deposit by changing the threshold value.

\subsubsection{The arrangemens}

The arrangement of the scintillator plate is shown in the Figure 3 and 4. The first arrangement is to place two plates parallel to the ground. The RI was placed in the middle between the plates. It was recorded when the particles passed through both plates. The second arrangement is to place a scintillator plate perpendicular to the ground. In this case, the effect of Co60 is almost equal to that of the first, and the amount of detection by background noise will be reduced. 


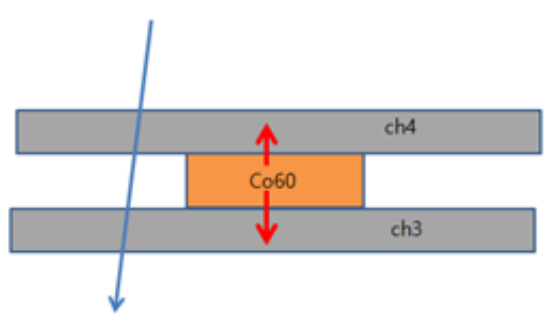

Figure 3: The horizontal arrangement. Blue arrow shows background noise like muon and red arrow shows gamma ray of Co60.

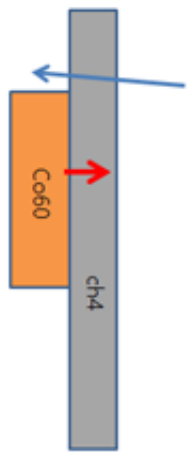

Figure 4: The vertical arrangement.

\section{Analysis}

\subsection{Analysis on the waveform}

The waveform of an event is recorded as shown in the following Figure 5. The analog signal is digitized in 1024 steps and the time is digitized to $950 \mathrm{~ns}$ in $10 \mathrm{~ns}$ increments by an $100 \mathrm{MHz}$ oscillator. The waveform appears in the form of a negative pulse. At this time, the area of the peak part of the waveform becomes a relative value proportional to the energy deposit. The upper part of the graph is integrated using the divisional quadrature method to obtain the area. The red box part of the Figure 5 is the pedestal value.

The peak occurs mostly within $100 \sim 200 n s$. Due to the characteristics of the PMT circuit, overshooting occurs immediately after the peak. Since the x-axis is 95 step, but the y-axis is 1024 steps, the whole range integration causes serious problems. The problem is that overshooting and peak are offset each other. The case when the event that the peak does not actually occur and the value that the small energy deposit generate are both 0 because of overshooting. To solve this problem, the integration range of the peak is set to $0 \sim 100 \mathrm{~ns}$. In order to consider the pedestal, the range of $750 \sim 950$ ns was integrated and the half value was defined as a pedestal at each event. This criterion allowed us to distinguish between null values and peaks.

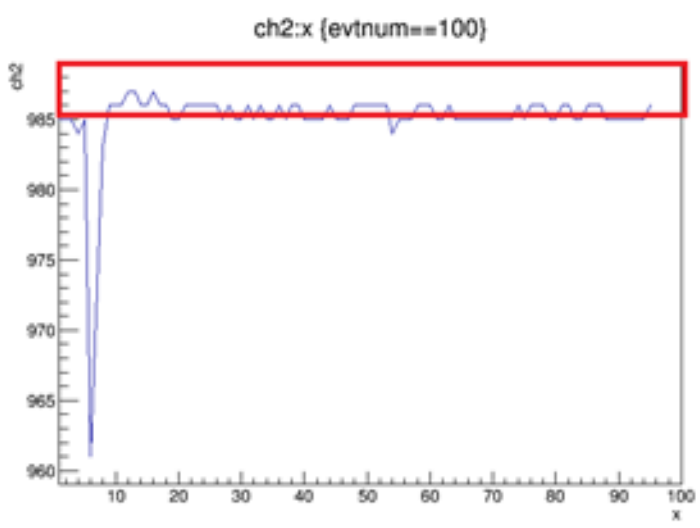

Figure 5: A waveform sample of an event passing through the scintillator 


\subsection{Geant4 simulation}

Geant4[5] was used for the detector simulation. The polystyrene scintillators were layered and filled with air between them. As primary particles, gamma of $1.17 \mathrm{MeV}$ and $1.33 \mathrm{MeV}$ which can occur in Co60 case was used. At this time, the energy deposit value obtained for each plate is used, and the number of events can be obtained for each trigger mode assuming a threshold value. Trigger mode indicates how many of the two plates exceeded the threshold.

\section{Result}

\subsection{Result of simulation}

The results obtained by the simulation are shown in the Figure 6 and 7. A gamma ray incident perpendicular to the scintillator leaves a distinct compton edge. Depending on the value of the primary energy, the range of the compton edge also changes. Due to the thickness of the plate, it seems that the full peak of the primary energy is difficult to detect. For $1.17 \mathrm{MeV}$, the compton edge occurs in the range of $0.8 \mathrm{MeV}$ to $1 \mathrm{MeV}$. For $1.33 \mathrm{MeV}$, the compton edge occurs between $1 \mathrm{MeV}$ and $1.2 \mathrm{MeV}$.

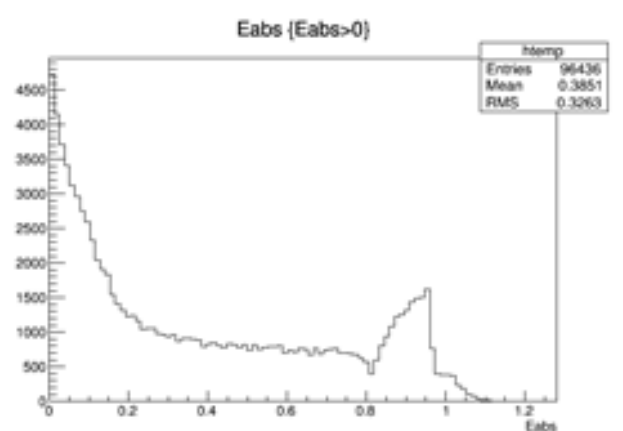

Figure 6: Simulated energy deposit of $1.17 \mathrm{MeV}$ gamma ray in $1 \mathrm{~cm}$ thick polystrene scintillator

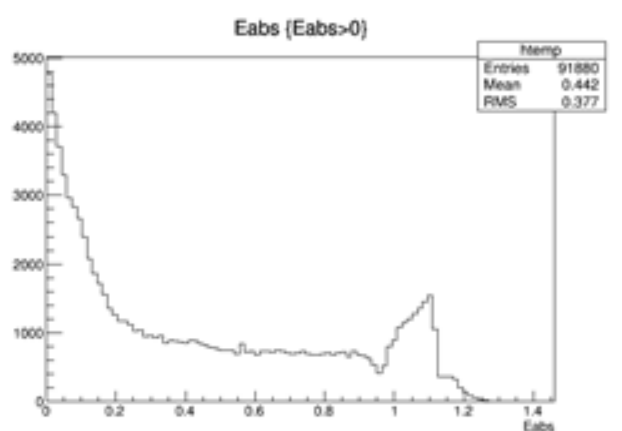

Figure 7: Simulated energy deposit of $1.33 \mathrm{MeV}$ gamma ray in $1 \mathrm{~cm}$ thick polystrene scintillator

\subsection{Distribution of energy according to scintillators arrangements}

\subsubsection{The horizontal arrangement}

The waveform integration value when the scintillator was disposed horizontally was examined. The red line in the Figure 8,9 and 10 is the result of Co60 and the blue line is the result of observing without Co60. When Co60 was placed, it was thought that the effect of the gamma ray emitted from the Co60 and the effect of the muon which is the background noise might coexist. The difference between two distributions may be due to gamma radiation emitted from Co60. The difference between two distributions is shown in the lower graph (Figure 8,9 and 10). In the upper graph of the Figure 8,9 and 10, the distribution of the background noise event normalized to the maximum value so as not to exceed the Co60 case. In order to consider the effect of the threshold value, various thresholds were tested. The values from the left are 955, 960, and 965. The threshold value is any value from 0 to 1024 , and the larger the number, the smaller the energy because of negative 


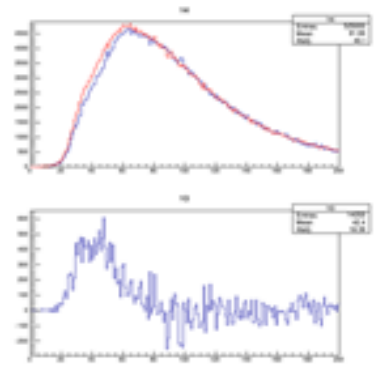

Figure 8: Relative value of energy deposit in the upper plate for the horizontal arrangement experiment with 955 threshold. Upper figure is the case with Co60(Red line) and the case without Co60(Blue line). Lower figure shows difference between two distributions.

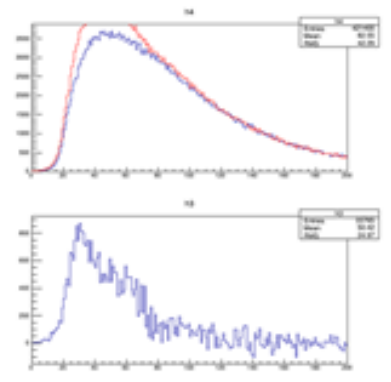

Figure 9: Relative value of energy deposit in the upper plate for the horizontal arrangement experiment with 960 threshold. Upper figure is the case with Co60(Red line) and the case without Co60(Blue line). Lower figure shows difference between two distributions.

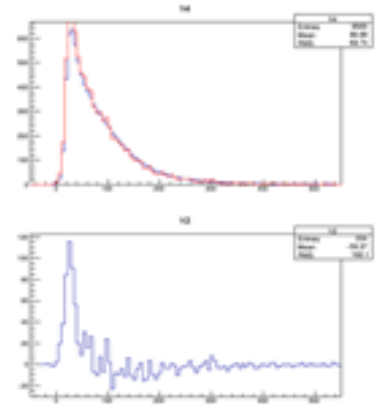

Figure 10: Relative value of energy deposit in the upper plate for the horizontal arrangement experiment with 965 threshold. Upper figure is the case with Co60(Red line) and the case without Co60(Blue line). Lower figure shows difference between two distributions.

pulse shape. When the threshold value is loose, the background is detected excessively, and when the threshold value is tight, it is impossible to detect the low energy gamma source. Therefore, we had to find the appropriate threshold for lower background ratio and higher source detection. In the Figure 8,9 and 10, it can be confirmed qualitatively that the threshold value of about 960 meets the above condition as compared with 955 or 965 . However, it is not easy to identify the Compton edge.

\subsubsection{The vetical arrangement}

Co60 was placed on the side of the plate to keep the effect of Co60 and to exclude the effect of background noise as much as possible. The red line in the Figure 11 is the result of the experiment in which Co60 is placed, and the blue line is the result of the experiment in which no cobalt is placed. The Figure 12 shows the difference between the two distributions, which can be thought of as the effect of the gamma rays generated in Co60. Depending on the normalization factor, the distribution in the Figure 12 can vary.

\subsection{Result of the fitting}

The Figure 13 shows the case for the normalization factor adjusted so that the number of events in the Figure 14 is not negative. By fitting this distribution using Dirac function, the Compton edge can be obtained. For this distribution, 79.37 FADC counts corresponds to $0.96 \mathrm{MeV}$, which is the compton edge. In the same distribution, the value corresponding to the backscattering can be obtained, and the full peak is defined as two parts where the height of the distribution is tall in a range larger than the compton edge. The values are shown in the Table 2.

It is the result of fitting the Table 2 with a linear function. The FADC count value can be converted to an energy value using the above results. It can be converted to $15.3 \mathrm{keV}$ per grid. 


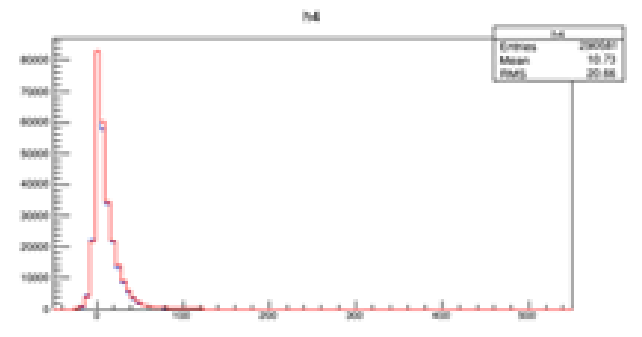

Figure 11: Distributions of relative value of energy deposit for the vertical arrangement experiment with 980 threshold. There are the case with Co60(Red line) and the case without Co60(Blue line).

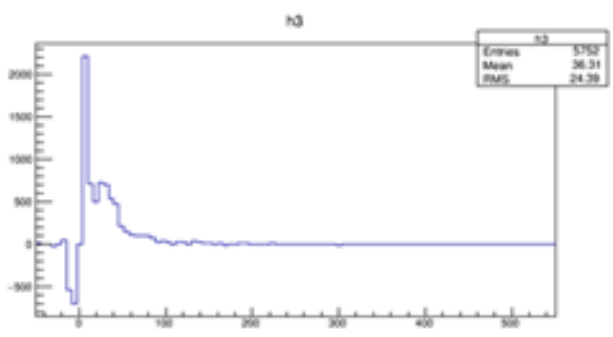

Figure 12: Difference between two distributions of Figure 11

\begin{tabular}{cc}
\hline Relative value(FADC counts) & Energy deposit $(\mathrm{MeV})$ \\
\hline 26 & 0.213 \\
79.4 & 0.96 \\
90 & 1.17 \\
94 & 1.25 \\
98 & 1.33 \\
\hline
\end{tabular}

Table 2: Relative values(FADC counts) and energy deposit $(\mathrm{MeV})$

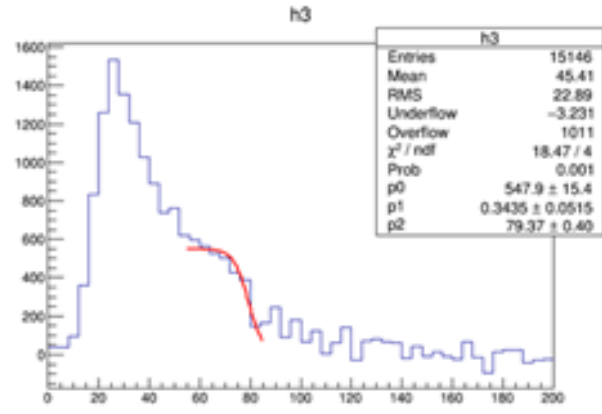

Figure 13: Fitting for the Compton edge

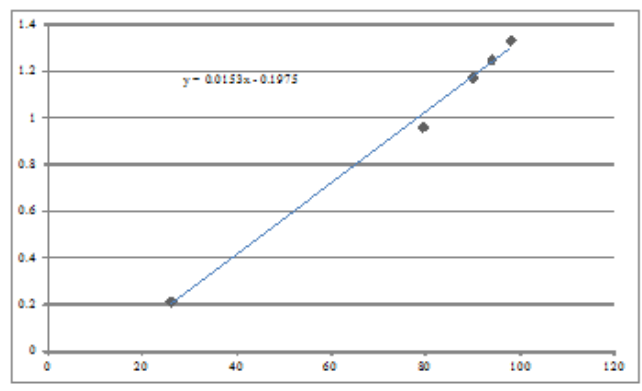

Figure 14: Relation between energy $\operatorname{deposit}(\mathrm{MeV})$ relative value(FADC counts)

\section{Discussion}

As a result of the above analysis, we could solve the waveform overshooting problem by setting the peak focused integral range. In addition, the difficulty of quantification in the form of a very thin and wide plate of $1 \mathrm{~cm}$ thickness and the background noise when is not shielded can be overcome to some extent through the arrangement of detectors. The results did not include error studies, but we could get a sketch of $15.3 \mathrm{keV}$ per grid. Using this, we expect to be able to quantify the energy of the cosmic rays and muons that are detected. Furthermore, when comparing two or more plates, it is expected that particle types can be discriminated. As a follow-up study, Geant 4 detector simulations are planned for gamma ray emission angle adjustment, background shielding experiments using lead bricks and data growth. In the analysis, the calculation of the 
systematic error caused by the normalization factor and the processing of the part of full peaks will be additionally performed for precise analysis.

\section{Summary}

$1 \mathrm{~cm}$ thick polystyrene scintillators are used to detect low energy cosmic rays. We tried calibration using Co60 to find out the absolute value of the energy deposit of cosmic rays. By adjusting the integral range, overshooting problems due to PMT circuit characteristics are solved. In addition, it was able to compensate the background problem by arranging vertically the plate scintillators. The characteristics of the compton edge were obtained through the Geant 4 simulation, and energy and the relative values obtained by integrating the waveforms were compared through fitting. A value of $15.3 \mathrm{keV}$ per grid was obtained. This will be used to compare the energy deposit of the muons, protons, and electrons in our experiments after the follow-up studies for quantitative analysis.

\section{References}

[1] W. Cho et al., Air Shower Events of High Energy Cosmic Rays Measured at Seoul, South Korea, AIP Conference Proceeding 1367, 232 (2011) [http: / / dx. doi.org/10.1063/1.3628753].

[2] J. Lee, COREA school array development for the measurement of extensive cosmic ray air shower and the study of air shower simulations, Master's thesis, Ewha Womens University(2007)

[3] S. Ashrafi et al., Energy calibration of thin plastic scintillators using Compton scattered $\gamma$ rays, Nucl. Instr. and Meth. A 642, 70 (2011) [https: / / doi.org/10.1016/j.nima.2011.04.003].

[4] S. Hohara et al., A simple method of energy calibration for thin plastic scintillator, IEEE TRANSACTIONS ON NUCLEAR SCIENCE 48, 4 (2001) [DOI : $10.1109 / 23.958745]$.

[5] GEANT4, Detector simulation tool home page, Available from http://http://geant4.cern.ch 